\title{
RESPONSABILIDAD SOCIAL EMPRESARIAL EN LA DIMENSIÓN LABORAL: CASO BANCOLOMBIA Y BBVA*
}

\author{
YENNI VIVIANA DUQUE OROZCO** \& DEISSY MARTÍNEZ BARÓN*** \\ UNIVERSIDAD MILITAR NUEVA GRANADA
}

Recibido/ Received/ Recebido: 26/01/2012 - Aceptado/ Accepted / Aprovado: 30/04/2012

\section{Resumen}

\begin{abstract}
Se construyó una matriz interpretativa que permitió evidenciar las prácticas en el ámbito laboral tomando como referente las propuestas por el Suplemento sobre el Sector de Servicios Financieros del Global Reporting Initiative- GRI. Los resultados muestran en ambos casos una fuerte preocupación en los aspectos de formación, salud y seguridad laboral; sin embargo, la brecha salarial entre hombres y mujeres, y la inexistente participación de las minorías son aspectos que aún no han superado. Las diferencias entre las dos entidades radican en las relaciones empresa- trabajadores y los beneficios sociales directos en forma de contribución monetaria.

Palabras clave: responsabilidad social empresarial, prácticas de responsabilidad social, sector bancario, dimensión laboral.

\section{ENTERPRISE SOCIAL RESPONSIBILITY IN LABORAL DIMENSION: CASE OF BANCOLOMBIA AND BBVA}

\begin{abstract}
An interpretative matrix was built in order to evidence labor practices based on proposals made by the Annex on Financial Services Sector of the Global Reporting Initiative-GRI. The results show that there is a strong preoccupation in training, health and labor security; however, the wage gap among men and women, and the inexistent participation of minority groups are aspects that have not been overcome. Differences among both cases are related to enterprise - worker relations and direct social benefits reflected in a monetary contribution.
\end{abstract}

Keywords: corporate social responsibility, social responsibility practices, banking sector, labor dimension.

* Artículo avance de investigación del proyecto ECO 565 "Prácticas de Responsabilidad Social Empresarial del Sector Bancario en Colombia", financiado por la Universidad Militar Nueva Granada. Las autoras agradecen la colaboración del pasante en investigación Daniel Bustos Garnica.

** Administradora de Empresas, especialista en Gerencia de la Calidad y Magíster en Gestión de Organizaciones de la Universidad Militar Nueva Granada. Docente de tiempo completo y miembro del Grupo de Emprendimiento y Empresariado- GEEMP- de la Universidad Militar Nueva Granada (Bogotá, Colombia). Correo electrónico: yenni.duque@unimilitar.edu.co

*** Economista y profesional en Relaciones Internacionales y Ciencias Políticas de la Universidad Militar Nueva Granada, estudiante de maestría en Economía en la Pontificia Universidad Javeriana. Auxiliar de Investigación del Grupo de Investigación en Emprendimiento y Empresariado- GEEMP- de la Universidad Militar Nueva Granada (Bogotá, Colombia). Correo electrónico: deissy.martínez@ unimilitar.edu.co 


\title{
RESPONSABILIDADE SOCIAL EMPRESARIAL NA DIMENSÃO TRABALHISTA: CASO BANCOLOMBIA E BBVA
}

\begin{abstract}
Resumo
Construiu-se uma matriz interpretativa que permitisse evidenciar as práticas no âmbito trabalhista tomando como referente as que foram propostas pelo Suplemento sobre o Setor de Serviços Financeiros do Global Reporting Initiative - GRI. Os resultados mostram que em ambos os casos há uma forte preocupação nos aspectos de formação, saúde e segurança trabalhista. No entanto, a brecha salarial entre homens e mulheres e a inexistente participação das minorias são aspectos que ainda não foram superados. As diferenças entre as duas entidades estão nas relações empresa - trabalhadores e nos benefícios sociais diretos em forma de contribuição monetária.

Palavras chave: responsabilidade social empresarial, práticas de responsabilidade social, setor bancário, dimensão trabalhista

Duque, Y. \& Martínez, D. (2012) Responsabilidad Social Empresarial en la dimensión laboral: caso Bancolombia y BBVA. En: Revista de la Facultad de Ciencias Económicas de la Universidad Militar Nueva Granada. rev.fac.cienc.econ, XX (1)

JEL: M14, J15, J16, J30, J50.
\end{abstract}

\section{Introducción}

En los últimos años la banca se ha caracterizado por obtener grandes rendimientos y, así mismo, no se han hecho esperar los cuestionamientos acerca de su papel en el campo de la RSE. El sector bancario es una de las fuentes más importantes de empleos formales directos en el país, impulsor económico de los demás sectores y fuente de inversión y crecimiento de la economía nacional, en otras palabras, se han convertido en motor de desarrollo económico y social del país.

En un entorno caracterizado por una población con necesidades básicas insatisfechas, difícil acceso a la educación, la salud y la vivienda, empleados mal remunerados, entre otras tantas carencias, es necesario que las organizaciones empresariales fijen y cumplan objetivos no sólo de carácter económico, sino también sociales y ambientales, a la vez que armonizan las relaciones con sus grupos de interés tanto externos como internos (Schvarstein, 2003, 45). Al respecto Fajardo $(2010,8)$ plantea que:

"En Colombia los grandes desequilibrios sociales, la exclusión social, los indicadores de concentración de ingresos en unos pocos, los niveles de desocupación, la carencia de espacios para el diálogo, los aumentos de las tasas de miseria e informalidad [...] constituyen argumentos sólidos para que los empresarios, los directivos de todas las organizaciones, toda la sociedad se auto-organice y ejecute acciones de compromiso institucional frente a la responsabilidad social".

En el ámbito laboral el sector bancario ha sido blanco de duras criticas, centradas en áreas como las bajas tasas de inclusión laboral de minorías, personas discapacitadas, pocas estrategias de prevención de riesgos laborales y promoción de salud y seguridad ocupacional (Alvarado, 2010; Asobancaria, 2005). Dado lo anterior, el presente documento busca analizar las prácticas de RSE en la dimensión laboral llevadas a cabo por Bancolombia y BBVA, caracterizados por ser dos de los bancos con mayor generación de utilidades en el país, cada uno en su categoría: nacional $e$ internacional, respectivamente.

Según la Superintendencia Financiera (2011), Bancolombia obtuvo utilidades en el año 2010 por un valor de 1,4 billones de pesos, registrando un crecimiento anual de 14,3 por ciento, lo que le permite mantener el liderazgo con respecto a sus competidores. Por otro lado, el BBVA es el banco de procedencia internacional que registra las mayores utilidades, reportando 420.000 millones de pesos, durante 
el año 2010 superando a Citibank. En el análisis se pretende identificar las prácticas de RSE en la dimensión laboral, y las diferencias relevantes entre los dos bancos.

La identificación y análisis de las prácticas de RSE, se hará a partir de una revisión documental de los Balances Sociales 2009 y 2010 (Bancolombia, 2009, 2010; BBVA, 2009, 2010) e información publicada en las páginas Web de las entidades a la luz del Suplemento Financiero del GRI. La selección del GRI como marco referencial para identificar las prácticas de RSE se da porque se fundamenta en orientaciones emitidas por la Organización Internacional del Trabajo (OIT), la Organización de las Naciones Unidas (ONU) y la Organización para la Cooperación Económica y el Desarrollo (OECD). Y por otro lado, porque este marco realizó adaptaciones propias para el sector financiero, el cual responde a las dinámicas y naturaleza de este tipo de entidades.

El documento contiene cinco secciones. En la primera, se presentan unos breves antecedentes del concepto de RSE, los aspectos a considerar en la dimensión laboral y una síntesis de los resultados que han arrojado los estudios que se han realizado específicamente en el sector bancario a nivel nacional e internacional. En la segunda parte se hace una reseña del sector bancario en Colombia y se presentan unos antecedentes de los bancos objeto de estudio. En el tercer apartado, se presenta la metodología y en el cuarto se analizan las prácticas de RSE en la dimensión laboral de los dos bancos. Finalmente, se exponen las conclusiones de los casos estudiados y algunas propuestas para la agenda de investigación.

\section{RSE, la dimensión laboral y el sector Bancario}

\subsection{Antecedentes del concepto de RSE}

La responsabilidad social empresarial, es un tema que ha sido ampliamente estudiado por diversas escuelas académicas, en tanto que en la literatura encontramos diversos conceptos al respecto. A continuación, presentaremos algunas de las definiciones de los autores más representativos en el campo de la RSE.
Uno de los primeros aportes académicos fue el de Bowen $(1953,6)$ quien la definió como "las obligaciones de los empresarios para aplicar políticas, tomar decisiones o seguir las líneas de acción que son deseables en términos de los objetivos y valores de nuestra sociedad". Una definición frecuentemente citada y mencionada ha sido la de Davis (1973), en la que enfrenta la posición libertaria de Friedman (1970), afirmando que "es obligación de la empresa evaluar en su procedimiento de toma de decisión los efectos de sus decisiones sobre el sistema social externo de una forma que logre beneficios sociales junto con los aumentos económicos tradicionales que la empresa busca. Significa que la responsabilidad social comienza donde la ley termina". En las definiciones expuestas se evidencian dos elementos claves; el primero relacionado con el receptor de dicha responsabilidad, y el segundo, con respecto al punto de partida de la responsabilidad de las empresas.

En los primeros trabajos se identifica un "sentido amplio" al reconocer a la sociedad como receptor de la responsabilidad de la empresa, es decir, la responsabilidad social implica que la empresa tenga en cuenta las demandas sociales en general y logre beneficios a la misma, a la vez que alcanza sus objetivos económicos. Por otro lado, a partir de lo propuesto por Davis en 1973, los conceptos de RSE dejan claro que ésta debe superar el carácter legal o normativo, autores como AECA (2003); Carroll (1979); Comisión de la Comunidad Europea (2001); Jones (1980); Raufflet (2010); Schvarstein (2003); McWilliams, Siegel \& Wright (2005); consideran en sus definiciones que la RSE requiere ir más allá de lo legal, lo que implica un carácter voluntario de la empresa con unos resultados no solo económicos.

Posteriormente, autores como Carroll (1991), Epstein (1987), Freeman (1984) y Schvarstein (2003), incluyen al concepto de RSE el término "stakeholders, planteando que la empresa debe tener en cuenta los intereses de las partes interesadas y armonizar sus relaciones con cada uno de ellos, ya no sólo con los stockholders o accionistas, como lo propuso Friedman (1970).

Al respecto, Freeman \& Reed $(1983,91)$ definen a los stakeholders como "cualquier grupo o individuo 
que pueda afectar o ser afectado por el logro de los propósitos de una corporación". Sin embargo, Bell $(1965,23)$ ya había dicho que "los accionistas son solamente un componente del conjunto de stakeholders y por ende, la organización asume una responsabilidad social más amplia, dirigida al bienestar de todos los involucrados en la organización y a la sociedad en la cual desarrolla sus actividades". Este nuevo elemento en el campo de la RSE hace que se particularice el ejercicio de la misma, es decir, que se identifiquen y responda a los intereses de los grupos con los que se relaciona la empresa.

Cortina (1997) en su libro "Ética de la empresa" hace referencia a los grupos de interés internos y externos de la empresa. Dentro de los internos considera a los socios y accionistas, directivos y trabajadores; $y$ en los externos a los clientes, proveedores, competidores, comunidad, entorno, Estado y sociedad en general. Sin embargo, los stakeholders o grupos de interés para las empresas son diversos y varían de acuerdo a su actividad económica o función dentro de la sociedad, y por supuesto a sus propósitos económicos, sociales y ambientales.

A finales de los setenta y comienzos de los ochentas autores como Zenisek $(1979,362)$ ya involucraban dentro de sus conceptos de RSE el término ética, para este autor la responsabilidad social corporativa es conceptuada como "el grado de ajuste entre las expectativas de la sociedad de la comunidad empresarial y la ética del negocio". Ese ajuste según el autor tiene dos componentes: el de comportamiento y el actitudinal. El primero tiene que ver con la satisfacción de las demandas sociales por parte de la empresa y el segundo con la ética de la misma, para el autor, actitudes directivas en cuanto a cuáles son o no son comportamientos socialmente responsables de la organización.

En la misma línea encontramos autores como Yepes, Peña \& Sánchez (2007), quienes reconocen a la articulación y a la autonomía como elementos claves de la RSE, estos dos, se manifiestan en los mismos términos que los propuestos por Zenisek (1979). Sin embargo, Yepes et al. (2007) desarrolla ampliamente estas características en términos que permiten establecer el tipo de prácticas que llevan a cabo las em- presas en diferentes medios sociales determinados por el nivel de cohesión social.

Dentro de este marco también ha de considerarse a Drucker $(1984,10)$ quien sugiere que la responsabilidad social es convertir un problema social en una oportunidad económica, productiva o laboral. A fin a esta propuesta, aunque más desarrollada, Porter \& Kramer (2006. 15) hablan de la creación de valor compartido como el "beneficio tanto para la sociedad como para la propia competitividad de la empresa"; cabe resaltar que la propuesta de estos autores ha sido ampliamente reconocida.

En conceptos más recientes, es decir, aquellos que aparecen a partir de los años 2000 aproximadamente, se aprecia -el cuidado del medio ambiente- como un elemento fundamental en la RSE (Abreu \& Badii, 2008; Comisión de las Comunidades Europeas, 2001; Martínez, Juanatey \& Silva, 2010; Sabogal, 2008) sugiriendo una búsqueda del equilibrio entre lo económico, lo social y lo ambiental.

Teniendo en cuenta todo lo anterior, vale la pena mencionar el trabajo realizado por el profesor Sabogal (2008) en el que encuentra los elementos comunes de los conceptos más relevantes de la RSE y construye una definición que los contiene, dando como resultado la siguiente propuesta:

"La RSE corresponde con un camino estratégico que busca crear valor a las empresas, generando relaciones beneficiosas en el largo plazo; son iniciativas de carácter voluntario y que por lo tanto deben superar los requerimientos legales y normativos; involucra una sostenibilidad que comprende tres elementos: el económico, el social y el ambiental; implica responsabilidad total para con todas las partes interesadas o stakeholders de la empresa." (Sabogal, 2008, 189).

\subsection{La RSE y la dimensión laboral}

En diferentes conceptualizaciones se menciona la necesidad de que la empresa asuma conductas éticas frente a sus empleados y que redunden en el 
mejoramiento de su calidad de vida y calidad en el trabajo. En el apartado anterior se señaló a Cortina (1997) para ilustrar la diferenciación entre: stakeholders internos y externos; en el primer grupo uno de los actores a considerar son los empleados, quienes de manera general tienen unos intereses como el pago de salarios justos, seguridad en el puesto de trabajo, promoción profesional y humana, y capacidad de participación.

Dicho lo anterior, es pertinente también mencionar lo dicho por Baltera \& Díaz $(2005,107)$ quien considera que la aplicación de la RSE en el ámbito interno constituye un paso adelante en el cumplimiento de la ley, y requiere un esfuerzo por "invertir en las personas, por incorporarlas a las decisiones, al menos en aquéllas que las afectan directamente y, por cierto, en las definiciones de políticas y estrategias internas de responsabilidad social".

A propósito, el Libro Verde realizado por la Comisión de las Comunidades Europeas $(2001,8)$ señala que: "Dentro de la empresa, las prácticas responsables en lo social afectan en primer lugar a los trabajadores y se refieren a cuestiones como la inversión en recursos humanos, la salud y la seguridad, y la gestión del cambio".

Por otro lado, organismos multilaterales como la OIT, la ONU, la OCDE han velado por el respeto $y$ por unas condiciones de trabajo dignas, convirtiéndose en referentes para analizar si efectivamente las prácticas de RSE de las empresas obedecen a las directrices emitidas por este tipo de organizaciones. Por ejemplo, la OIT (1998) habla de cuatro principios mínimos del derecho al trabajo: (i) el respeto por la libertad de asociación y el reconocimiento efectivo del derecho de negociación colectiva; (ii) la eliminación del trabajo forzado u obligatorio; (iii) la abolición del trabajo infantil; y (iv) la eliminación de cualquier discriminación con respecto al empleo.

El GRI (2008) en su categoría de Prácticas Laborales y Trabajo Digno considera 5 aspectos principales: (i) empleo; (ii) relaciones empleo/trabajadores; (iii) salud y seguridad laboral; (iv) formación y evaluación; y (v) diversidad e igualdad de oportunidades. Los indica- dores de desempeño que contempla cada aspecto se orientan por las directrices de la Organización Internacional del Trabajo (OIT), la Organización para la Cooperación Económica y el Desarrollo (OECD) y la Organización de las Naciones Unidas (ONU).

\subsection{La RSE en el sector bancario}

En la revisión realizada de los artículos científicos publicados en los últimos trece años acerca de la RSE en el sector bancario $y / o$ financiero se encontraron 47 documentos, y la mayoría de ellos se concentran en estudiar la divulgación de la RSE a través de sus páginas Web, sus formas, a quién la dirigen y en otros casos, pretenden medir su impacto en los clientes; algunos ejemplos son: Farook \& Lanis (2005), Castelo \& Lima (2008), Khan, et al. (2009), Hassan \& Syafri (2010), Hinson, et al. (2010). Con respecto a la RSE en los bancos los estudios muestran de forma general lo siguiente:

- Confusión conceptual de la RSE con mecenazgo o filantropía empresarial (Sarro, et al., 2007).

- La RSE es un aspecto creciente e importante en la industria bancaria internacional. Hay una asociación positiva y significativa entre la RSE de los bancos, y su calidad y tamaño financiero (Sholtens, 2009).

- La adaptación sectorial de la iniciativa GRI ha propiciado el aumento en el número de adhesiones no sólo a esta iniciativa sino a otras de nivel internacional del sector (Ruiz, et al., 2009).

- Los bancos cada vez más usan el marco GRI y el protocolo técnico para el sector financiero con relación a lo reportado, esto muestra que el GRI está asistiendo a los bancos para que la RSE sea más consistente y sea un factor de cambio para el rendimiento de RSE (Viganò \& Nicolai, 2006).

- Una gran proporción de la sociedad sigue siendo excluida financieramente. La mayoría de veces la RSE está lejos de la conciencia corporativa de los bancos, por lo general se queda en planes y agendas (Chambers \& Day, 2009).

- La política gubernamental es un factor importante en el diseño del contexto que modela la mayoría de las acciones tomadas por el sector bancario (Jeucken \& Bouma, 1999). 
- El contenido de la información RSE es detallada pero no hay evidencia de los resultados obtenidos (Ásványi, 2009).

Aunque no hay estudios que indaguen específicamente acerca de la RSE interna de los bancos, hay algunos resultados que se rescatan:

- Las compañías cada vez más reportan en sus actividades de RSE, personal, políticas de desarrollo y procesos para manejar la RSE y crear responsabilidades de gestión y dirección en el área (Humphreys \& Brown, 2008).

- Los bancos (kenianos) no divulgan información relevante concerniente a convocatorias, empleo de grupos especiales, asistencia a empleados pensionados, productividad de los empleados, entre otras (Barako \& Brown, 2008).

- No hay relación significativa entre la representación femenina en la junta directiva y el reporte de RSE (Narwal, 2007).

- Una buena práctica con respecto al género es la implementación a largo plazo de la igualdad de género como un elemento indispensable de toda estrategia de RSE para todas las empresas. Los instrumentos de gestión de RSE internos son importantes para alcanzar un resultado a nivel de la compañía y los instrumentos externos son indispensables para alcanzar impactos en la sociedad (Schultz, 2007).

Para el contexto colombiano los estudios de RSE en el sector bancario son escasos, tan solo se encontraron tres aproximaciones, de los cuales dos son trabajos de grado uno para optar por el título de pregrado (Pinilla, et al., 2006) y el otro por título de maestría (Montes, 2008), y una ponencia (Niño \& Llorente, 2009). Sin embargo, vale la pena resaltar algunos hallazgos relevantes:

- $\quad$ "Las entidades con un mayor grado de madurez en temas de responsabilidad social, perciben que el aumento de la penetración de los servicios financieros básicos es parte de sus compromisos con la sociedad. Además se tiene el indicio de que estas entidades tienen una mayor facilidad para diseñar estrategias novedosas que permiten incrementar el acceso del servicio a la población de bajos recursos" (Niño \& Llorente, 2009).

- "Para asegurar la sostenibilidad en el largo plazo tanto para los establecimientos bancarios en Colombia como para la sociedad y el ambiente, las dimensiones ambiental, económica, social e institucional son fundamentales para el análisis y aplicación de la Ecoeficiencia y la RSE" (Montes, 2008).

En esta sección vale la pena resaltar la orientación del gremio representativo del sector financiero en Colombia Asobancaria en lo que respecta a la RSE en la dimensión laboral. En el Balance Social (2010, 7) de esta entidad y en palabras de su presidenta hace referencia a que uno de los aportes más importantes de la banca ha sido: "la generación de empleo, pilar esencial de la responsabilidad social de las entidades", discurso que se queda corto en estos tiempos donde se comprende que la generación de empleo más que una acción voluntaria es un requerimiento para poder desarrollar la actividad económica de cualquier organización.

\section{Reseña del sector bancario en Colombia}

La banca comercial inicia en la década del 70 con la creación de las Corporaciones de Ahorro que tenían por objeto apoyar proyectos masivos de construcción, política promovida por el gobierno Pastrana de 1970 a 1974 (Rodríguez \& Tovar, 2007). Los primeros bancos comerciales privados constituidos a nivel nacional fueron manejados por la clase alta del país, la cual estaba concentrada en Bogotá, no obstante, existían bancos privados en los Estados Federales quienes manejaban su propia emisión de billetes.

Luego se crearon entidades bancarias que pertenecían al sector público dado que promovían y apoyaban los diversos sectores económicos, pero al mismo tiempo competían con los bancos en el sector privado por sus actividades financieras. Posteriormente, se fue incrementando la participación en el sector financiero de más entidades bancarias; sin embargo, el sector bancario ha sufrido alteraciones que han requerido una reconfiguración en su estructura dada las dinámicas de la apertura económica, y 
unas de las más importantes han sido las fusiones y adquisiciones de entidades bancarias, con el fin de mantenerse y fortalecerse en el mercado, así como para contribuir a la competitividad del sector a nivel nacional y extranjero.

Las múltiples fusiones e integraciones en el sector financiero de las dos últimas décadas han reconfigurado el panorama constituyendo grandes grupos financieros. Actualmente, la Superintendencia Financiera (2010) tiene adscritas veintiuna (21) entidades bancarias como establecimientos de crédito al 2010, estas son: Banco de Bogotá, Banco Popular, Banco Santander, Bancolombia, The Royal Bank of Scotland (Colombia) S.A., Banco Citibank, HSBC Colombia S.A., Banco GNB Sudameris S.A., Banco Bilbao Vizcaya Argentaria BBVA Colombia S.A., Helm Bank S.A., Banco de Occidente, Banco Caja Social BCSC, Banco Davivienda, Red Multibanca Colpatria, Banagrario, Aliadas, Av. Villas, Banco ProCredit Colombia S.A., Banco de las Microfinanzas - Bancamía S.A.

Los grupos bancarios más importantes que hacen parte del sistema financiero en la actualidad se pueden ver en la tabla 1.

Tabla 1. Grupos Financieros en Colombia $^{1}$

\begin{tabular}{|c|c|}
\hline Grupos Financieros & Bancos que los conforman \\
\hline Grupo Bancolombia & Bancolombia \\
\hline \multirow{2}{*}{ Grupo Aval } & Banco de Bogotá \\
\cline { 2 - 2 } & Banco de Occidente \\
\cline { 2 - 2 } & Banco Popular \\
\hline $\begin{array}{c}\text { Grupo Banco Bilbao Vizcaya } \\
\text { Argentaria (Colombia) }\end{array}$ & Banco AvVillas \\
\hline \multirow{2}{*}{ Grupo BCSC } & BBVA \\
\cline { 2 - 2 } & Banco Caja Social \\
\hline Grupo Santander & Banco Colmena \\
\hline
\end{tabular}

Las fusiones y adquisiciones en la banca dejan como grandes participantes al Grupo AVAL, Davivienda,
Bancolombia y BBVA, y así mismo lo confirman los resultados económicos de estas entidades en los últimos años. Estos autores le atribuyen este hecho a las fuertes barreras de ingreso a otros participantes, liderazgo total en costos y generación de economías de escala (Rodríguez \& Tovar, 2007; Ortega, 2006)

Actualmente, el sector bancario está regulado por la Superintendencia Financiera de Colombia, la cual fue el resultado de la fusión entre la Superintendencia Bancaria y la Superintendencia de Valores. Frente a esta entidad, los bancos deben presentar una serie de requerimientos que garanticen el cumplimiento de la ley en la realización de las actividades propias de naturaleza financiera y bancaria.

Por su parte, la Superintendencia Financiera ha promovido aspectos como la adopción de Códigos de Buen Gobierno y realiza anualmente la Encuesta Código País, la cual, busca determinar aspectos relevantes sobre la adopción de las recomendaciones del Código de Mejores Prácticas Corporativas de Colombia.

De igual forma, Asobancaria ha promovido la adopción e implementación de las prácticas de RSE mediante la realización de una encuesta a bancos y corporaciones financieras con el fin de identificar las experiencias de las entidades al realizar su balance social. La encuesta consta de tres partes principales: gestión socialmente responsable, servicios prestados $y$, actividad de intermediación y demografía, empleo $y$ relaciones laborales.

Actualmente, tan solo tres bancos en Colombia están adheridos al Pacto Global y cuatro a los Principios de Ecuador, ver tabla 2. El Pacto Global es una iniciativa voluntaria en la que las empresas se comprometen a integrar en sus estrategias y operaciones diez principios universalmente aceptados en cuatro aspectos: derechos humanos, estándares laborales, medio ambiente y anti-corrupción. Por otra parte, los Principios de Ecuador son unos lineamientos estándar del sector financiero para determinar, evaluar $y$ administrar el riesgo socio-ambiental en la financiación de proyectos.

\footnotetext{
1 Fuente: Las autoras con base en información de la Superintendencia Financiera de Colombia.
} 
Tabla 2. Bancos en Colombia adheridos al Pacto Global y/o Principios de Ecuador ${ }^{2}$

\begin{tabular}{|l|c|c|}
\hline \multicolumn{1}{|c|}{ Banco } & Pacto Global & $\begin{array}{c}\text { Principios de } \\
\text { Ecuador }\end{array}$ \\
\hline BBVA Colombia & 2002 & 2004 \\
\hline Bancolombia (Grupo) & 2008 & 2008 \\
\hline Banco Santander & No adherido* & 2009 \\
\hline Citigroup & No adherido* & 2003 \\
\hline $\begin{array}{l}\text { Bancoomeva (Coomeva } \\
\text { Cooperativa Financiera) }\end{array}$ & 2011 & No adherido \\
\hline
\end{tabular}

\subsection{Antecedentes Bancolombia}

En 1998 el Banco de Colombia propiedad de la familia Gilinski y otros accionistas minoritarios, y el Banco Industrial Colombiano (BIC) del sindicato antioqueño se fusiona, para convertirse en Bancolombia. Posteriormente, en el año 2005 Bancolombia se fusiona con Conavi y Corfinsura, con el fin de enfrentar los retos de la globalización en el mercado financiero. De esta manera se diversificó el portafolio de servicios de la entidad, se amplió la cobertura de los mismos y se fortaleció financieramente, siendo hoy el banco privado más grande del país y con una fuerte participación en el mercado (ver tabla 3).

Bancolombia tiene presencia en el exterior con filiales en Panamá, Puerto Rico, Islas Caimán, Perú y cuenta con una agencia en Miami. Los principales accionista de Bancolombia son el Grupo de Inversiones Suramericana con el 29\%, los Fondos de Pensiones con un 19,7\% y American Depositary Receipts con el 19,6\% (Grupo de Inversiones Suramericana, 2011).

Recientemente, fue catalogada como la empresa del mercado financiero de América Latina con mejor manejo de las redes sociales (premio otorgado por la revista Global Finance). Así mismo, fue premiada como la mejor sucursal virtual para personas en
Colombia con una política basada en la actitud, asesoría y calidad (Revista Enter, 2011).

En el primer semestre de 2011 Bancolombia registró una variación en su utilidad neta de $16,4 \%$ con respecto al mismo periodo en el 2010. El monto de sus activos para junio de 2011 asciende a $\$ 75,156$ billones de pesos, y su patrimonio a $\$ 8,007$ billones de pesos constituyéndolo en el banco más grande del país (Grupo de Inversiones Suramericana, 2011).

Tabla 3. Participación en el Mercado de Bancolombia ${ }^{3}$

\begin{tabular}{|l|c|}
\hline \multicolumn{1}{|c|}{ Producto } & \% Participación \\
\hline Total Cuenta Corriente & 23,5 \\
\hline Total CDT & 14,2 \\
\hline Total Ahorros & 20,1 \\
\hline Total Depósitos & 19,1 \\
\hline Cartera Neta & 21,0 \\
\hline
\end{tabular}

\subsection{Antecedentes Banco Bilbao Vizcaya Argentaria (BBVA) Colombia}

En 1998 se fusiona el Banco Bilbao y el Banco de Vizcaya para crear BBV y a finales de 1999 se fusiona éste con el Banco Argentaria estableciendo la marca BBVA. Como consecuencia de lo anterior, este grupo adquiere un tamaño importante, fuerte solvencia patrimonial y gran estructura financiera, convirtiéndose en uno de los bancos más fuertes de España.

En el proceso de expansión del BBV, en 1996 adquieren el Banco Ganadero de Colombia fundado en 1956 por un grupo de ganaderos del país, el cual tenía por objeto impulsar y fomentar la actividad agropecuaria y que para el momento de la adquisición el 68,84\% del capital estaba en poder de los accionistas particulares, el 29,68\% en manos del Gobierno y el $1,48 \%$ de la Caja Agraria.

2 Fuente: Las autoras con base en la información de la página oficial del Global Compact UN y Principios de Ecuador. Página Oficial Pacto Global: www.unglobalcompact.org/participant/search. Página Oficial Principios de Ecuador: http://www.equator-principles.com/ index.php/members-reporting/members-and-reporting (consultado 01 septiembre 2011). Las subsidiarias en Colombia no están adheridas.

3 Fuente: Grupo de Inversiones Suramericana (Información a junio 30 de 2011). 
El Banco Ganadero en 1994 coloco sus acciones en Wall Street y a mediados de 1996 el BBV adquirió el $40 \%$ de su capital accionario, y en 1998 adquiere un $15 \%$ adicional. Tras la fusión del BBV y el Banco Argentaria, se da una capitalización del BBVA lo que le permite ser el propietario del 85,12\% del capital accionario del Banco Ganadero. En el 2001 el BBVA incrementó su propiedad sobre el Banco Ganadero logrando el 95,16\% de las acciones.

El BBVA hoy tiene presencia en las principales economías de América Latina con inversiones de primer nivel en bancos, fondos de pensiones, aseguradoras, banca de inversión y otras actividades del sector financiero. Según datos de la Superintendencia Financiera a Junio de 2011, el BBVA es el cuarto banco más grande del país por sus activos ( $\$ 23,261$ billones de pesos COP) y el quinto por su patrimonio ( $\$ 2,289$ billones de pesos COP). De los bancos internacionales con presencia en el país es el más grande y el que obtiene mayores utilidades, ver tabla 4.

Tabla 4. Ranking de los Bancos Internacionales en Colombia ${ }^{4}$

\begin{tabular}{|c|l|c|}
\hline Posición & \multicolumn{1}{|c|}{ Banco } & $\begin{array}{c}\text { Resultados a Junio 2011 } \\
\text { (Millones de Pesos COP) }\end{array}$ \\
\hline 1 & BBVA & $229.826,03$ \\
\hline 2 & Citibank & $66.449,93$ \\
\hline 3 & Helm Bank & $62.459,09$ \\
\hline 4 & Santander & $61.245,25$ \\
\hline 5 & Sudameris & $55.790,63$ \\
\hline 6 & Scotia Bank & $-5.187,73$ \\
\hline 7 & Colombia & $-32.048,4$ \\
\hline
\end{tabular}

\section{Metodología}

Se desarrollo un análisis de contenido de los Informes Sociales (2009 y 2010) de los dos bancos. Se construyó una matriz interpretativa que permitiera evidenciar las prácticas en el ámbito laboral tomando como referente las propuestas por el Suplemento sobre el Sector de Servicios Financieros del Global Reporting Initiative- GRI, el cual considera las acciones particulares y la naturaleza del sector en las dimensiones: productos y servicios, económica, ambiental, y social. Dentro de la dimensión social consideran las prácticas laborales y éticas del trabajo, derechos humanos, sociedad y responsabilidad sobre productos.

Con respecto a las prácticas laborales el GRI considera cinco aspectos: empleo, relaciones empresatrabajadores, salud y seguridad en el trabajo, formación y educación, y diversidad e igualdad de oportunidades. En la tabla 5 se aprecian los indicadores considerados en cada uno de los aspectos.

Otra de las razones por las cuales se toma como referente el Suplemento para el Sector de Servicios Financieros (SSSF) del GRI y, se explica porque los bancos estudiados presentan sus informes sociales bajo esta metodología, lo que permite una comparación de correspondencia entre lo que se expone en los informes y los indicadores del GRI.

Krippendorff $(1990,28)$ define el Análisis de Contenido como "la técnica destinada a formular, a partir de ciertos datos, inferencias reproducibles y válidas que puedan aplicarse a un contexto". Esta técnica permite analizar la naturaleza del discurso en cualquiera de sus formas de comunicación. En el presente trabajo se partió del análisis de fuentes secundarias, antes mencionadas, y se tomaron los datos tal $y$ como son comunicados a través de éstos, desde un enfoque cualitativo con el objeto de comparar los indicadores presentados en la tabla 5 y los reportados por los bancos en sus informes sociales, de tal forma que se evidencie la correspondencia entre éstos y los reportados por las entidades financieras estudiadas. Lo anterior permitió tomar como categorías los cinco aspectos de la dimensión laboral referenciados en la tabla 5 .

\footnotetext{
4 Fuente: Las autoras con base en la Información de la Superintendencia Financiera a Junio 2011.
} 
Tabla 5. Indicadores de Prácticas Laborales del GRI para el sector financiero 5

\begin{tabular}{|c|c|}
\hline Aspectos & Indicadores \\
\hline Empleo & $\begin{array}{l}\text { LA1 Desglose del colectivo de trabajadores por tipo de } \\
\text { empleo, por contrato y por región. } \\
\text { LA2 Número total de empleados y rotación media de } \\
\text { empleados, desglosados por grupo de edad, sexo y } \\
\text { región. } \\
\text { LA3 Beneficios sociales para los empleados con jor- } \\
\text { nada completa, que no se ofrecen a los empleados } \\
\text { temporales o de media jornada, desglosado por ac- } \\
\text { tividad principal. }\end{array}$ \\
\hline $\begin{array}{l}\text { Relaciones } \\
\text { empresa/traba- } \\
\text { jadores }\end{array}$ & $\begin{array}{l}\text { LA4 Porcentaje de empleados cubiertos por un con- } \\
\text { venio colectivo. } \\
\text { LA5 Periodo(s) mínimo(s) de preaviso relativo(s) a } \\
\text { cambios organizativos, incluyendo si éstas notifica- } \\
\text { ciones son especificadas en los convenios colectivos. }\end{array}$ \\
\hline $\begin{array}{l}\text { Salud y Se- } \\
\text { guridad en el } \\
\text { Trabajo }\end{array}$ & $\begin{array}{l}\text { LA6 Porcentaje del total de trabajadores que está re- } \\
\text { presentado en comités de salud y seguridad conjuntos } \\
\text { de dirección-empleados, establecidos para ayudar a } \\
\text { controlar y asesorar sobre programas de salud y segu- } \\
\text { ridad en el trabajo. } \\
\text { LA7 Tasas de absentismo, enfermedades profesio- } \\
\text { nales, días perdidos y número de víctimas mortales } \\
\text { relacionadas con el trabajo por región. } \\
\text { LA8 Programas de educación, formación, asesora- } \\
\text { miento, prevención y control de riesgos que se apli- } \\
\text { quen a los trabajadores, a sus familias o a los miem- } \\
\text { bros de la comunidad en relación con enfermedades } \\
\text { graves. } \\
\text { LA9 Asuntos de salud y seguridad cubiertos en acuer- } \\
\text { dos formales con sindicatos. }\end{array}$ \\
\hline $\begin{array}{l}\text { Formación y } \\
\text { Educación }\end{array}$ & $\begin{array}{l}\text { LA10 Promedio de horas de formación al año por em- } \\
\text { pleado, desglosado por categoría de empleado. } \\
\text { LA11 Programas de gestión de habilidades y de for- } \\
\text { mación continúa que fomenten la empleabilidad de } \\
\text { los trabajadores y que les apoyen en la gestión del } \\
\text { final de sus carreras profesionales. } \\
\text { LA12 Porcentaje de empleados que reciben evalua- } \\
\text { ciones regulares del desempeño y de desarrollo pro- } \\
\text { fesional. }\end{array}$ \\
\hline $\begin{array}{l}\text { Diversidad e } \\
\text { Igualdad de } \\
\text { Oportunidades }\end{array}$ & $\begin{array}{l}\text { LA13 Composición de los órganos de gobierno cor- } \\
\text { porativo y plantilla, desglosado por sexo, grupo de } \\
\text { edad, pertenencia a minorías y otros indicadores de } \\
\text { diversidad. } \\
\text { LA14 Relación entre salario base de los hombres con } \\
\text { respecto al de las mujeres, desglosado por categoría } \\
\text { profesional. }\end{array}$ \\
\hline
\end{tabular}

\section{RSE en la dimensión laboral en Bancolombia y BBVA}

\subsection{Características generales del empleo en Bancolombia y el BBVA}

En lo relativo al empleo se considera relevante la estabilidad, los beneficios sociales y la inversión en el equipo humano como indicadores que permiten identificar las prácticas laborales aplicadas para cumplir la estrategia de las entidades. Según González \& Arias (2011), durante el año 2010, el sector bancario incorporó por contrato a término indefinido a más del $88 \%$ de sus empleados. Del total de la plantilla, el $94 \%$ trabajan tiempo completo y el $47 \%$ llevan más de 5 años en la entidad. En cuanto a capacitación las entidades otorgaron más de \$21 mil millones de pesos en créditos y subsidios.

Con respecto a la variable de empleo se analiza la amplitud y diversidad del colectivo de trabajadores de la entidad bancaria, principalmente por tipo de contrato, edad y sexo. Al respecto las dos entidades presentan un crecimiento de su plantilla; sin embargo, ambas se abstienen de comunicar acerca de la distribución de su plantilla por tipo de contrato y rotación de los empleados, por lo tanto, no se aprecia la estabilidad laboral que brindan estas corporaciones a sus colaboradores.

En el mismo aspecto se consideran también los beneficios sociales que brinda la entidad a los empleados de planta. En este indicador se consideran los beneficios sociales directos en forma de contribución monetaria, se excluyen entonces los beneficios en especie, tales como actividades deportivas, servicio gratuito de alimentación, etc. En este frente se evidencia una diferencia entre las dos corporaciones, de acuerdo a la información reportada. Bancolombia ofrece beneficios sociales como: tasas preferenciales en diferentes líneas de crédito, salud complementaria, seguros y fondo mutuo; en tanto el BBVA no reporta información al respecto (ver tabla 6).

5 Fuente: Suplemento sobre el Sector de Servicios Financieros del Global Reporting Initiative- GRI. 
Tabla 6. Matriz de Análisis del Indicador de Empleo ${ }^{6}$

\begin{tabular}{|c|c|c|c|}
\hline Aspecto & Indicador & Bancolombia & BBVA \\
\hline \multirow{3}{*}{ EMPLEO } & $\begin{array}{l}\text { LA1 } \\
\text { Desglose del colecti- } \\
\text { vo de trabajadores. }\end{array}$ & $\begin{array}{l}\text { - Presenta un crecimiento de su plantilla. } \\
\text {-No hay información desagregada del tipo de con- } \\
\text { trato (termino fijo o indefinido) de la plantilla de } \\
\text { la entidad. } \\
\text {-Con respecto a la distribución de empleados por } \\
\text { región, la entidad tiene operaciones en Antioquia, } \\
\text { Bogotá, Caribe, centro y sur del país. Siendo An- } \\
\text { tioquia y Bogotá las zonas con mayor oferta labo- } \\
\text { ral ( } 41,2 \% \text { y } 25 \% \text { respectivamente). }\end{array}$ & $\begin{array}{l}\text {-Presenta un crecimiento de su plantilla. } \\
\text {-No hay información desagregada del tipo de contrato } \\
\text { (termino fijo o indefinido) de la plantilla de la entidad. } \\
\text {-Con respecto a la distribución de empleados por re- } \\
\text { gión, la entidad tiene operaciones en } 23 \text { regiones del } \\
\text { país; sin embargo, el } 55 \% \text { de los empleos ofrecidos se } \\
\text { concentran en la capital. } \\
\text {-Se registra un incremento en su plantilla del } 32,14 \% \text {, } \\
\text { pasando de } 4.284 \text { empleados en el } 2009 \text { a } 5.661 \text { en } \\
\text { el } 2010 \text {, la edad promedio se mantiene constante en } \\
37 \text { años. }\end{array}$ \\
\hline & $\begin{array}{l}\text { LA2 } \\
\text { Rotación. }\end{array}$ & $\begin{array}{l}\text {-Presentan el promedio de tiempo de permanen- } \\
\text { cia de los empleados en la corporación ( } 8,6 \text { años } \\
\text { para mujeres y 10,7 años para hombres). } \\
\text {-No reporta la rotación media de los empleados, } \\
\text { ni cuantos dejaron su empleo en el año objeto del } \\
\text { informe. Por lo tanto, no se tienen datos de la ro- } \\
\text { tación por edad, sexo y región. }\end{array}$ & $\begin{array}{l}\text {-No hay información con respecto a la rotación media } \\
\text { de empleados. Hay desglose del total de los emplea- } \\
\text { dos por rangos de edad y categoría profesional, pero } \\
\text { no se encuentra desagregada la rotación media de em- } \\
\text { pleados por edad, región y sexo. } \\
\text {-El rango de antigüedad de los empleados se concen- } \\
\text { tra entre } 1-5 \text { años con un } 23.3 \%, 10-20 \text { años con un } \\
36,2 \% \text {. } \\
\text {-Se evidencia un descenso del } 50 \% \text { en la antigüedad } \\
\text { promedio de los trabajadores pasando de } 12,3 \text { a } 6,2 \\
\text { años. El } 24 \% \text { de esa diferencia se explica por el ingreso } \\
\text { de los nuevos colaboradores (1.377), mientras que se } \\
\text { infiere que el } 26 \% \text { restante es explicado por despidos } \\
\text { de personas que exceden cierto tiempo de antigüedad, } \\
\text { jubilación, entre otras. }\end{array}$ \\
\hline & $\begin{array}{l}\text { LA3 }^{*} \\
\text { Beneficios sociales } \\
\text { a los empleados de } \\
\text { Tiempo Completo. }\end{array}$ & $\begin{array}{l}\text {-Tasas preferenciales para líneas de crédito: vi- } \\
\text { vienda, vehículo, calamidad, educación y consu- } \\
\text { mo (las tasas son menores en casi un } 40 \% \text { con } \\
\text { respecto a las tasas de los clientes). El informe } \\
\text { muestra un crecimiento en los créditos para vi- } \\
\text { vienda y vehículo y un descenso en el resto, con } \\
\text { respecto al año } 2009 \text { y } 2008 \text {. Más de } 33 \% \text { de su } \\
\text { plantilla fue beneficiada en el año } 2010 \text {. } \\
\text {-Contribución monetaria a los planes comple- } \\
\text { mentarios de salud (más del } 60 \% \text { de sus emplea- } \\
\text { dos tienen estos planes). } \\
\text {-Seguros de automóviles, vida (patronal), hábitat. } \\
\text {-Fondo mutuo. }\end{array}$ & $\begin{array}{l}\text {-No hay información con respecto a algún beneficio } \\
\text { social directo en forma de contribución monetaria a los } \\
\text { empleados. }\end{array}$ \\
\hline
\end{tabular}

\subsection{Relaciones empresa-trabajadores}

En el aspecto de relaciones empresa-trabajadores las dos entidades bancarias cuentan con sindicatos, en donde más del $60 \%$ de sus empleados hacen parte de algún tipo de convención y/o pacto colectivo; sin embargo, en lo que respecta a garantizar el diálogo sobre los cambios organizativos significativos, Bancolombia no reporta información y el BBVA declara que no tienen establecido un tiempo mínimo de

6 Fuente: Las autoras con base en la información de los informes de Responsabilidad Social de las entidades. (*) Estos indicadores corresponden a los adicionados en el suplemento sectorial financiero del GRI. 
preaviso. De acuerdo a lo establecido por el GRI establecer un tiempo de preaviso para los cambios organizativos ayuda a minimizar los impactos adversos a los empleados, esta práctica ayuda a mantener un ambiente laboral sano, reduciendo la rotación, entre otras. Lo anterior se puede evidenciar en la tabla 7.

Con respecto al BBVA, fuentes secundarias como documentos de los sindicatos, ponen de manifiesto la falta de oportunidades de ascenso de los trabajadores sindicalizados y políticas que debilitan los sindicatos a través de la promoción de un pacto colectivo (Silverman, 2006; ACEB, 2011). Mientras que Bancolombia ha sido ejemplo en los procesos de diálogo y negociación sindical, con miras al mejoramiento del clima laboral y construcción de confianza (Fajardo, 2010).

Tabla 7. Matriz de Análisis del Indicador de Relaciones Empresa/Trabajadores ${ }^{7}$

\begin{tabular}{|c|c|c|c|}
\hline Aspecto & Indicador & Bancolombia & BBVA \\
\hline \multirow[t]{2}{*}{$\begin{array}{l}\text { RELACIONES EMPRE- } \\
\text { SA/ TRABAJADORES }\end{array}$} & $\begin{array}{l}\text { LA4 } \\
\text { Convenios colectivos. }\end{array}$ & $\begin{array}{l}\text {-La convención colectiva vigente cubre cerca de } \\
11.115 \text { empleados, cuyos cargos se encuentran in- } \\
\text { cluidos en el escalafón de cargos. } \\
\text { operativos, es decir, el } 69 \% \text { de la comunidad laboral } \\
\text { del Banco. }\end{array}$ & $\begin{array}{l}\text {-Más del } 76,7 \% \text { de los empleados pertenecen } \\
\text { al pacto colectivo; el } 22,5 \% \text { hace parte del sin- } \\
\text { dicato. }\end{array}$ \\
\hline & $\begin{array}{l}\text { LA5 } \\
\text { Preaviso a cambios } \\
\text { organizativos. }\end{array}$ & -No hay información en el reporte. & $\begin{array}{l}\text {-No hay información en el reporte y para el } \\
\text { informe del } 2010 \text { afirman no tener definido un } \\
\text { tiempo mínimo de preaviso. }\end{array}$ \\
\hline
\end{tabular}

\subsection{Salud y seguridad en el trabajo}

Al respecto de la salud y seguridad en el trabajo una de las prácticas a considerar son los comités de seguridad y salud con representación tanto de los empleados como de los niveles directivos, dado que el involucramiento de los colaboradores en este proceso mejora este aspecto en los puestos de trabajo. En los informes de ambas corporaciones se reportan actividades de salud y seguridad ocupacional, dentro de las que se encuentran programas de prevención de enfermedades, accidentes, y emergencias, evaluaciones médicas e identificación de riesgos: sin embargo, no se hace mención a los comités existentes ni a la participación de los empleados, sus familias o la comunidad en los mismos. En la tabla 8 se aprecia el análisis de los indicadores del presente aspecto.

\subsection{Formación y educación}

La categoría de formación y educación es, sin duda uno de las prácticas más intensas en el plano laboral. Ambas entidades registran actividades de formación a sus empleados que les permita su cualificación y mejoramiento de habilidades. Se presenta una diferencia en ambas entidades y tiene que ver con la formación interna y externa. El BBVA se concentra en formación interna, mientras que Bancolombia lo hace tanto interno como externo, es decir, genera oportunidades de formación en el exterior. Sin embargo, la información suministrada no permite conocer el grado en el que se distribuye la formación y educación en las diferentes categorías profesionales; tampoco se evidencian prácticas en lo que respecta a promover el desarrollo de capacidades de los empleados que están pronto a jubilarse o a ser des-

7 Fuente: Las autoras con base en la información de los informes de Responsabilidad Social de las entidades. (*) Estos indicadores corresponden a los adicionados en el suplemento sectorial financiero del GRI. 
Tabla 8. Matriz de Análisis del Indicador de Salud y Seguridad en el Trabajo ${ }^{8}$

\begin{tabular}{|c|c|c|c|}
\hline Aspecto & Indicador & Bancolombia & BBVA \\
\hline \multirow{4}{*}{$\begin{array}{l}\text { SALUD Y SE- } \\
\text { GURIDAD EN EL } \\
\text { TRABAJO }\end{array}$} & $\begin{array}{l}\text { LA6* } \\
\text { Comités de salud y seguridad } \\
\text { laboral. }\end{array}$ & $\begin{array}{l}\text { - Reportan programas de salud laboral, pero no } \\
\text { se mencionan los comités formales de seguri- } \\
\text { dad y salud ni la representación conjunta de la } \\
\text { dirección y de los empleados en los comités } \\
\text { de seguridad y salud ocupacional, como lo } \\
\text { indica el GRI. }\end{array}$ & $\begin{array}{l}\text { - Reportan programas de salud laboral, pero no } \\
\text { se menciona la representación conjunta de la } \\
\text { dirección y de los empleados en los comités de } \\
\text { seguridad y salud ocupacional, como lo indica } \\
\text { el GRI. }\end{array}$ \\
\hline & $\begin{array}{l}\text { LA7 } \\
\text { Datos de seguridad y salud en } \\
\text { el trabajo. }\end{array}$ & $\begin{array}{l}\text {-Presentan algunas actividades realizadas con } \\
\text { respecto a la seguridad y salud en el trabajo; } \\
\text { sin embargo, no reporta datos de accidentes } \\
\text { laborales, absentismo, enfermedades profe- } \\
\text { sionales, etc. }\end{array}$ & $\begin{array}{l}\text {-Presenta algunos datos de salud y seguridad } \\
\text { en el trabajo; sin embargo, no se desglosan por } \\
\text { ciudad, ni por tipo de contrato. Se incremento el } \\
\text { número de accidentes del año } 2009 \text { al } 2006 \text { en } \\
\text { un poco más del } 6 \% \text {. Otra variable que aumentó } \\
\text { fue el número de enfermedades profesionales } \\
\text { que paso de } 26 \text { a } 55 \text {. }\end{array}$ \\
\hline & $\begin{array}{l}\text { LA8 } \\
\text { Educación, formación asesora- } \\
\text { miento a empleados, sus fami- } \\
\text { lias y comunidad con respecto } \\
\text { a enfermedades. }\end{array}$ & $\begin{array}{l}\text {-Tienen programas de salud y seguridad la- } \\
\text { boral; sin embargo, no se menciona que en } \\
\text { el programa se involucre a sus familias o a la } \\
\text { comunidad. Tampoco hay datos del alcance y } \\
\text { cobertura de estos programas. }\end{array}$ & $\begin{array}{l}\text {-Tienen un programa de salud y seguridad laboral } \\
\text { que promueve los estilos de vida saludable; sin } \\
\text { embargo, no se menciona que en el programa se } \\
\text { involucre a sus familias o a la comunidad. }\end{array}$ \\
\hline & $\begin{array}{l}\text { LA9* } \\
\text { Salud y seguridad cubiertos en } \\
\text { acuerdos formales con sindi- } \\
\text { catos. }\end{array}$ & -No hay información en los reportes. & -No hay información en los reportes. \\
\hline
\end{tabular}

pedidos, con el fin de asegurar su empleabilidad o transición a su nuevo estatus laboral. Ninguna de las dos entidades reporta el proceso de evaluación de desempeño a sus empleados. En la tabla 9 se exponen los hallazgos del indicador de formación y educación.

\subsection{Diversidad e igualdad de oportunidades}

Por último, en lo que concierne a la diversidad $e$ igualdad de oportunidades, la plantilla del BBVA muestra una participación mayor por parte de los hombres en todas las categorías profesionales, excepto en la fuerza de ventas. Las brechas de participación se intensifican en las categorías: directivos y directivos corporativos (mujeres $0,54 \%$-hombres $1,28 \%$ y mujeres $0,03 \%$ - hombres $0,40 \%$ respecti- vamente). En la misma entidad la distribución de los salarios promedio por sexo en las diferentes categorías profesionales son mayores para los hombres. En el nivel de equipo directivo la brecha entre los salarios promedio de los hombres y las mujeres asciende al $13,4 \%$. No hay información en cuanto a la participación de las minorías en el equipo de colaboradores de la entidad.

Bancolombia en cuanto a la diversidad e igualdad de oportunidades no reporta prácticas, lo único que se abstrajo de los reportes es la participación femenina en el nivel de gobierno corporativo en el cual solo participa 1 mujer entre 23 integrantes (4,3\%). En la tabla 10 se evidencian los resultados del análisis al indicador de diversidad e igualdad de oportunidades.

8 Fuente: Las autoras con base en la información de los informes de Responsabilidad Social de las entidades. (*) Estos indicadores corresponden a los adicionados en el suplemento sectorial financiero del GRI. 
Tabla 9. Matriz de Análisis de Indicadores de Formación y Educación ${ }^{9}$

\begin{tabular}{|c|c|c|c|}
\hline Aspecto & Indicador & Bancolombia & BBVA \\
\hline \multirow{3}{*}{$\begin{array}{l}\text { FORMACIÓN Y } \\
\text { EDUCACIÓN }\end{array}$} & $\begin{array}{l}\text { LA10 } \\
\text { Promedio de horas de formación al año } \\
\text { por empleado, desglosado por catego- } \\
\text { ría de empleado. }\end{array}$ & $\begin{array}{l}\text {-Se registró un incremento en el año } 2010 \\
\text { del } 19,19 \% \text { en la participación de los em- } \\
\text { pleados en capacitaciones presenciales y } \\
\text { de un } 68,3 \% \text { virtuales. El informe reporta } \\
\text { un promedio de } 8 \text { cursos por empleado; } \\
\text { sin embargo, los datos suministrados en } \\
\text { esta sección no se presentan de acuerdo a } \\
\text { los lineamientos del GRI, lo que no permite } \\
\text { identificar. } \\
\text {-Los datos suministrados al respecto de la } \\
\text { formación no permiten identificar el grado } \\
\text { en que se distribuye entre la totalidad de la } \\
\text { plantilla. }\end{array}$ & $\begin{array}{l}\text {-Se evidencia del } 2009 \text { al } 2010 \text { un creci- } \\
\text { miento del } 7 \% \text { en la participación en ca- } \\
\text { pacitaciones y que se le da relevancia a la } \\
\text { categoría profesional de Fuerza de Ventas. } \\
\text { Es importante aclarar que no presentan el } \\
\text { indicador como lo sugiere el GRI y que } \\
\text { los datos en la sección de capacitación y } \\
\text { formación no coindicen con otros referen- } \\
\text { ciados en el mismo reporte y no permiten } \\
\text { reconocer los empelados por categoría } \\
\text { profesional capacitados. Tampoco es claro } \\
\text { el cálculo del dato de cursos promedio por } \\
\text { empleado }(6,5) \text {. }\end{array}$ \\
\hline & $\begin{array}{l}\text { LA11* } \\
\text { Programas de habilidades } \\
\text { y de formación continua que fomenten } \\
\text { la empleabilidad de los trabajadores y } \\
\text { de los próximos a jubilarse. }\end{array}$ & $\begin{array}{l}\text {-Formación interna y externa. } \\
\text {-Becas Excelencia Bancolombia tiene como } \\
\text { objetivo ofrecer formación avanzada en el ex- } \\
\text { terior a empleados de destacado desempeño } \\
\text { y con proyección para cargos críticos de la } \\
\text { organización (5 empleados en el } 2010 \text { - Aus- } \\
\text { tralia, España y Londres). } \\
\text { - No hay información con respecto a políticas } \\
\text { y programas dirigidos al desarrollo de capa- } \\
\text { cidades de los trabajadores que están próxi- } \\
\text { mos a jubilarse } 0 \text { a ser despedidos. }\end{array}$ & $\begin{array}{l}\text {-La información señalada en los informes } \\
\text { no específica financiación para formación o } \\
\text { educación externa o sí conceden períodos } \\
\text { de comisión de estudios, garantizando el } \\
\text { regreso al empleo. } \\
\text {-No hay información con respecto a políti- } \\
\text { cas y programas dirigidos al desarrollo de } \\
\text { capacidades de los trabajadores que están } \\
\text { próximos a jubilarse o a ser despedidos. }\end{array}$ \\
\hline & $\begin{array}{l}\text { LA12* } \\
\text { Evaluaciones del desempeño y de } \\
\text { desarrollo profesional. }\end{array}$ & -No hay información al respecto & -No hay información al respecto. \\
\hline
\end{tabular}

\section{Conclusiones}

Uno de los primeros hallazgos es que las entidades bancarias estudiadas, pese a que afirman que su marco de reporte social es el GRI, no están respondiendo a dichos indicadores o presentan información relativa, pero no la desagregan como lo indica la guía. La forma de presentación de los datos varia en los diferentes reportes por año, lo que impide una comparación entre los mismos.

El suplemento del GRI para el sector de servicios financieros sugiere que las entidades financieras reportan políticas y prácticas relacionadas a las amenazas y violencia en el lugar de trabajo, para apoyar a los empleados, sus familias o comunidad, en las circunstancias de ataques y agresiones por parte de clientes $\mathrm{u}$ otros, atracos, lavado de dinero o terrorismo. Frente a lo anterior no se evidencian programas de educación, prevención, orientación o control de riesgos.

Se identificó que ambas entidades le apuntan a la formación de sus empleados y a realizar actividades de salud y seguridad ocupacional; sin embargo, en aspectos como la igualdad de oportunidades es clara la existencia de una brecha salarial entre las mujeres y los hombres, se ofrecen mayores ingresos a los hombres pese a que las mujeres ocupan la misma

9 Las autoras con base en la información de los informes de Responsabilidad Social de las entidades. (*) Estos indicadores corresponden a los adicionados en el suplemento sectorial financiero del GRI. 
Tabla 10. Matriz de Análisis de Indicadores de Diversidad e Igualdad de Oportunidades ${ }^{10}$

\begin{tabular}{|c|c|c|c|}
\hline Aspecto & Indicador & Bancolombia & BBVA \\
\hline \multirow[t]{2}{*}{$\begin{array}{l}\text { DIVERSIDAD E } \\
\text { IGUALDAD DE } \\
\text { OPORTUNIDADES }\end{array}$} & $\begin{array}{l}\text { LA13 } \\
\text { Composición de los órganos de } \\
\text { gobierno corporativo y plantilla, } \\
\text { desglosado por sexo, grupo de } \\
\text { edad, pertenencia a minorías y } \\
\text { otros indicadores de diversidad. }\end{array}$ & $\begin{array}{l}\text {-No presentan la información con respecto } \\
\text { a la distribución de los empleados por ca- } \\
\text { tegorías profesionales. Por lo tanto, no se } \\
\text { aprecia la distribución de los empleados } \\
\text { por sexo (mujer/hombre), edad o minorías } \\
\text { en las diferentes categorías, ni de manera } \\
\text { global. } \\
\text {-Del análisis de la información se abstrae } \\
\text { que en el nivel de gobierno corporativo } \\
\text { solo participa } 1 \text { mujer entre } 23 \text { integrantes } \\
(4,3 \%) \text {. }\end{array}$ & $\begin{array}{l}\text {-La distribución de empleados por sexo (mujer/ } \\
\text { hombre) en las diferentes categorías profesionales } \\
\text { es principalmente masculina, excepto en la fuerza } \\
\text { de ventas. Las brechas se intensifican en las cate- } \\
\text { gorías: directivos y directivos corporativos ( } 0,54 \% \text { - } \\
1,28 \% \text { y } 0,03 \%-0,40 \% \text { respectivamente). } \\
\text {-La plantilla de empleados por grupos de edad estáa } \\
\text { concentrada en el rango } 30-50 \text { años ( } 64,42 \%) \text {, me- } \\
\text { nores de } 30 \text { ( } 26 \% \text { ), mayores a } 50 \text { ( } 9,8 \%) \text {. } \\
\text { - No presentan la información de participación por } \\
\text { edad en las diferentes categorías profesionales y } \\
\text { tampoco hay información al respecto de la partici- } \\
\text { pación de las minorías en la plantilla de empleados } \\
\text { de la corporación. }\end{array}$ \\
\hline & $\begin{array}{l}\text { LA14 } \\
\text { Relación entre salario base de } \\
\text { hombres / mujeres, desglosado } \\
\text { por categoría profesional. }\end{array}$ & -No hay información al respecto. & $\begin{array}{l}\text { - En todas las categorías profesionales sin excep- } \\
\text { ción, los hombres tienen un salario base mensual } \\
\text { mayor que el de las mujeres. En el nivel de equipo } \\
\text { directivo la diferencia asciende al } 13,4 \% \text {. } \\
\text { - No hay información con relación a la categoría de } \\
\text { directores corporativos. }\end{array}$ \\
\hline
\end{tabular}

categoría profesional. Por otro lado, tampoco se aprecia la participación de las minorías en este tipo de entidades.

Con respecto a las relaciones laborales empresa- trabajadores y a la libre asociación, la información divulgada en los documentos formales de las entidades consultados no es detallada, ni se presenta conforme a la metodología del GRI. Por lo tanto, no son claras las oportunidades de ascenso para los trabajadores sindicalizados y las políticas organizacionales de cara a la libre asociación. Es importante consultar fuentes primarias en tanto hay algunas fuentes secundarias, como por ejemplo documentos de los sindicatos, que manifiestan las políticas contrarias a este derecho por parte de entidades bancarias.

La calidad del empleo, las condiciones de trabajo, la conciliación entre la vida laboral y familiar, la equidad de género, la inclusión de grupos étnicos o vulnerables, la remuneración justa, las relaciones laborales y la libre asociación, entre otros, son los objetivos que se requieren sean cumplidos por parte de las entidades bancarias y las organizaciones en general.

Como se evidencia, la RSE del sector bancario no ha sido un tema suficientemente explorado en nuestro país, el cual se convierte en un espacio de estudio potencial para los investigadores. El papel de las instituciones financieras frente a las demandas sociales de países en desarrollo como el nuestro, el nivel de utilidades versus la inversión social (más allá de la legal), los resultados e impactos generados por las prácticas de RSE llevadas a cabo por las entidades bancarias, son espacios de investigación potencial que requieren ser explorados.

10 Fuente: Las autoras con base en la información de los informes de Responsabilidad Social de las entidades. (*) Estos indicadores corresponden a los adicionados en el suplemento sectorial financiero del GRI. 


\section{Referencias}

Abreu, J. \& Badii, M. (2008) "Análisis del concepto de responsabilidad social empresarial". En: Daena: International Journal of Good Consciencie, 2(1): 54-70.

Asociación Colombiana de Empleados Bancarios-ACEB (2011) "Comunicación de la ACEB a el BBVA (Incentivo EDU-EDI)". Disponible en: http://aceb.org.co/documentos/comunicacion_EDU-EDI_BBVA_marzo_16_2011.pdf

Alvarado, D. (2010) "Lo Décente én el Trabajo: ¿Responsabilidad Social, Moral o Política de la Empresa?". En: Observatorio Laboral Revista Venezolana, 3 (5): 111-141.

Asobancaria (2005) "Una mirada a la responsabilidad social de la banca colombiana y mundial". En: La semana económica de Asobancaria, edición No 523. Disponible en: http://www. asobancaria.com/upload/docs/docPub2754 2.pdf

Asobancaria (2010) "Balance Social de la Banca Colombiana 2010". Disponible en: http://www.asobancaria.com/portal/ page/portal/Asobancaria/responsabilidad social/balance social_del_sector_financiero/Balance\%20social\%20de\%20la\%20 banca\%202010.

Asociación Española de Contabilidad y Administración de Empresas- AECA (2003) "Marco Conceptual de la Responsabilidad Social Corporativa". Madrid: AECA.

Ásványi, K. (2009) CSR communication of a large bank. Proceedings of FIKUSZ '09 Symposium for Young Researchers, 17-28. Published by Budapest Tech Keleti Károly Faculty of Economics.

Baltera, \& Díaz, E. (2005) "Responsabilidad social empresarial alcances y potenciales en materia laboral". Gobierno de Chile, Dirección del trabajo, Departamento de estudios, Cuadernos de investigación, núm. 25. Disponible en: http://www.dt.gob. cl/1601/articles-88984_recurso_1.pdf

Bancolombia (2009) "Informe responsabilidad social empresarial Grupo Bancolombia". Disponible en: http://www.grupobancoIombia.com/contenidoCentralizado/informacionEmpresarial/ relacionInversionistas/gobiernoCorporativo/resultadosFinancieros/resultadosAnuales/anuales/2009/InformeSocialBancolombia2009.pdf

Bancolombia (2010) "Informe responsabilidad social empresarial. Grupo Bancolombia". Disponible en: http://www.grupobancolombia.com/contenidoCentralizado/informacionEmpresarial/ relacionlnversionistas/gobiernoCorporativo/resultadosFinancieros/resultadosAnuales/anuales/2010/InformeResCor_Abril2010. pdf

Barako, D. \& Brown, A. (2008) "Corporate social reporting and board representation: evidence from the Kenyan banking sector". En: Journal of Management \& Governance, 12 (4): 309-324.

BBVA (2009) "Informe responsabilidad social empresarial Banco Bilbao Viscaya Argentaria". Disponible en: http://www.bbva. com/TLBB/fbin/IARC09 Colombia tcm12-223593.pdf

BBVA (2010) "Informe responsabilidad social empresarial. Banco Bilbao Viscaya Argentaria". Disponible en: http://bancaparatodos.com/wp-content/uploads/BBVA-Colombia-IARC-2010.pdf

Bell, D. (1965) "The Corporation and Society in the 1970's". En: The Public Interest, 24: 5-32.

Bowen, H. (1953) Social Responsibilities of the Businessman. New York: Harper \& Row.

Carroll, A. (1979) "A three-dimensional conceptual model of corporate performance". En: Academy of Management Review, 4 (4): 497-505.
Carroll, A. (1991) "The pyramid of corporate social responsibility: toward the moral management of organizational stakeholders". En: Business Horizons, 34 (4): 39-48.

Castelo, M. \& Lima, L. (2008) "Social responsibility disclosure: A study of proxies for the public visibility of Portuguese banks". En: The British Accounting Review, 40: 161-181

Chambers, C. \& Day, R. (2009) "The banking sector and CSR: An unholy alliance?" En: Financial Regulation International, $12(9): 13-20$

Comisión de las Comunidades Europeas- CCE (2001) Libro Verde: Fomentar un marco europeo para la responsabilidad social de las empresas. Bruselas.

Cortina, A. (1997) Rentabilidad de la ética para la empresa. Visor: Fundación Argentaria.

Davis, K. (1973) "The case for and against business assumption of social responsibilities". En: Academy of Management Journal, 16 (2): 312-322.

Drucker, P. (1984), "The new meaning of corporate social responsibility". En: California Management, Review, 26 (2): 53-63.

Epstein, E. (1987) "The corporate social policy process: Beyond business ethics, corporate responsibility, and corporate social responsiveness". En: California Management Review, 29 (3): 99-114.

Freeman, R. (1984) Strategic Management: A Stakeholder Approach. New York.

Freeman, E. \& Reed, D. (1983) "Stockholders and Stakeholders: A New Perspective on Corporate Governance". En: California Management Review, 25: 88-106.

Friedman, M. (1970) "The social responsibility of business is to increase its profits". En: The New York Times Magazine, septiembre 13 de 1970: 1-6.

Fajardo, E. (2010) Responsabilidad social empresarial: una reflexión desde la gestión; lo social y ambiental. Bogotá: Universidad Nacional de Colombia, Facultad de Ciencias Económicas.

Farook, S. \& Lanis, R. (2005) "Banking on Islam? Determinants of corporate social responsibility disclosure". Accounting and Finance Association of Australia and New Zealand Conference, Melbourne, Australia, July 2005 in 2005 AFAANZ Conference Proceedings, ed Faff, R, AFAANZ, Melbourne, Australia, $p$ 1-45. Disponible en: http://islamiccenter.kau.edu.sa/arabic/ Ahdath/Con06/_pdf/Vol1/22\%20Sayd\%20Zubair\%20Farook\%20 Banking\%20on\%20lslam.pdf

González, M. \& Arias, P. (2011) "La Responsabilidad Social de la Banca". En: La semana Económica de Asobancaria, edición No 809. Disponible en: http://www.asobancaria.com/portal/ $\mathrm{pls} /$ portal/docs/1/1384048.PDF

Global Reporting Initiative -GRI (2008) "Guía para la elaboración de Memorias de Sostenibilidad y Suplemento Sectorial para Servicios Financieros (FSSS)". Disponible en: https://www. globalreporting.org/resourcelibrary/Spanish-FSSS-ReportingGuidelines.pdf

Grupo de Inversiones Suramericana (2011) "Portafolio de servicios". Disponible en: http://www.gruposuramericana.com/Sections/ NuestroPortafolio/Grupo\%20Bancolombia.aspx

Hassan, A. \& Syafri, S. (2010) "Exploring corporate social responsibility disclosure: the case of Islamic banks". En: International Journal of Islamic and Middle Eastern Finance and Management, 3 (3): 203-227.

Hinson, R.; Boateng, R. y Madichie, N. (2010) "Corporate social responsibility activity reportage on bank websites in Ghana". En: International Journal of Bank Marketing, 28 (7): 498-518. 
Humphreys, M. \& Brown, M. (2008) "An Analysis of Corporate Social Responsibility at Credit Line: A Narrative Approach". En: Journal of Business Ethics, 80: 403-418.

Jeucken, M. \& Bouma, J. (1999) "The Changing Environment of Banks". En: Greener Management International, 21-35.

Jones, T. (1980) "Corporate Social Responsibility Revisited, Redefined". En: California Management Review, 22 (3): 59-67.

Khan, M., Halabi, A. y Samy, M. (2009) "Corporate social responsibility (CSR) reporting: a study of selected banking companies in Bangladesh". En: Social Responsibility Journal, 5 (3): 344-357.

Krippendorff, K. (1990) Metodología del análisis de contenido. Teoría y Práctica. Barcelona. Paidós Ibérica, S.A

Martínez, V., Juanatey, O. y Silva, M. da, (2010) "Percepciones del concepto de la responsabilidad social corporativa: un estudio exploratorio". En: Hologramática, facultad de Ciencias Sociales (UNLZ), 3 (13): 3-34.

McWilliams, A.; Siegel, D. \& Wright, D. (2005) "Corporate Social Responsibility: Strategic Implications". En: Journal of Business Strategies (Rensselaer Working Papers in Economics), $N^{\circ}$ 0506: 1-31.

Montes, J. (2008) Ecoeficiencia: una propuesta de responsabilidad ambiental empresarial para el sector financiero colombiano. Tesis de maestría no publicada. Medellín: Universidad Nacional de Colombia, Facultad de Minas.

Narwal, M. (2007) "CSR Initiatives of Indian Banking Industry". En: Social Responsibility Journal, 3 (4):49-60.

Niño, D. \& Llorente, B. (2009) Equidad y eficiencia en la banca colombiana: evidencia del aporte de las prácticas de Responsabilidad Social Empresarial. Ponencia en IX Congreso latinoamericano ALENE. (Septiembre 24 y 25 de 2009).

Organización Internacional del Trabajo-OIT (1998) Declaración de la OIT relativa a los principios y derechos fundamentales en el trabajo y su seguimiento. Organización Internacional del Trabajo, Conferencia Internacional del Trabajo en su octogésima sexta reunión, Ginebra, Suiza. Disponible en: http:// www.cinterfor.org.uy/public/spanish/region/ampro/cinterfor/ publ/boletin/143/pdf/bol2.pdf

Ortega, M. (2006) "Cambios de género y discriminación laboral en el sector financiero colombiano. El caso de Bancolombia". En: AIBR Revista de Antropología Iberoamericana, 1 (3): 526-547.

Pinilla, D., Osorio, M. y Orozco, F. (2006) Responsabilidad social empresarial del sector financiero en colombia (con énfasis en el sector bancario). Tesis de pregrado no publicada. Bogotá: Pontificia Universidad Javeriana, Facultad de Derecho. Disponible en: http://www.javeriana.edu.co/biblos/tesis/derecho/ dere8/DEFINITIVA/TESIS05.pdf

Porter, M. \& Kramer, M. (2006) "Estrategia y sociedad". En: Harvard Business Review, 84 (12): 42-56.

Raufflet, E. (2010) "Responsabilidad de las empresas y desarrollo sostenible". En: Cuadernos de Administración, Universidad del Valle, (43): 23-32.

Revista Enter (2011) "Bancolombia: el mejor banco en redes sociales de Latinoamérica" Disponible en: http://www.enter.co/ otros/bancolombia-el-mejor-banco-en-redes-sociales-del-pais/
Rodríguez, C. \& Tovar, J. (2007) "Fusiones y adquisiciones como estrategia de crecimiento en el sector bancario Colombiano". En: Innovar Revista de Ciencias Administrativas y Sociales, Universidad Nacional de Colombia, 17 (30): 77-98.

Ruiz, M., Rios, A. y Tirado, P. (2009) "La responsabilidad social y la crisis económica. ¿Responden las entidades financieras españolas a los grupos de interés?". En: Revista de Economía Pública, Social y Cooperativa, (65): 33-58.

Sabogal, J. (2008), "Aproximación y cuestionamientos al concepto de responsabilidad social empresarial". En: Investigación y Reflexión, Universidad Militar Nueva Granada, XVI (1): 179-195.

Sarro, M.; Cuesta, ; Penelas A. (2007) "La responsabilidad social corporativa (RSC): una orientación emergente en la gestión de las entidades bancarias españolas" En: "Conocimiento, innovación y emprendedores: camino al futuro" / coord. por Juan Carlos Ayala Calvo (Universidad de La Rioja).

Schvarstein, L. (2003) La inteligencia social de las organizaciones. Argentina: Paidós.

Sholtens, B. (2009) "Corporate Social Responsibility in the International Banking Industry". En: Journal of Business Ethics, 86: $159-175$.

Shultz, I. (2007) "Corporate Social Responsibility and Gender Equality in the Banking Sector - a Case Study. En: Rhetoric And Realities: Analysing Corporate Social Responsibility in Europe". Disponible en: http://www.isoe.de/fileadmin/redaktion/ Downloads/Gender/rare-16-banking-2007.pdf

Superintendencia Financiera de Colombia (2010). "Resultados Establecimientos de Crédito 2010". Disponible en: http:// www.superfinanciera.gov.co/ComunicadosyPublicaciones/ establecimientos 122010.zip

Silverman, J. (2006) "Proyecto Observatorio Social de Empresas Transnacionales Españolas en Suramérica". Informe BBVA Colombia. Escuela Nacional Sindical Medellín, Colombia. Disponible en: www.ens.org.co/aa/img.../Informe_BBVA_proyecto ISCOD.doc

Superintendencia Financiera de Colombia (2011). "Actualidad del Sistema Financiero Colombiano 2011". Disponible en: ttp:// www.superfinanciera.gov.co/ComunicadosyPublicaciones/ comsectorfinanciero062011.pdf

Viganò, F \& Nicolai, D. (2006) "CSR in the European Banking Sector: Evidence from a Sector Survey". En: Rhetoric And Realities: Analysing Corporate Social Responsibility in Europe. Disponible en: http://www.rare-eu.net/fileadmin/user upload/ internal/project documents/Sector_Survey/RARE CSR Survey_-Banking_Sector.pdf

Yepes G., Peña, W. \& Sánchez, L. (2007) Responsabilidad Social Empresarial, Fundamentos y Aplicación en las organizaciones de hoy. Bogotá D.C., Universidad Externado de Colombia.

Zenisek, T. (1979) "Corporate social responsibility: A conceptualization based on organizational literature". En: Academy of Management Review, 4 (3): 359-368. 